\title{
Astrocytic Reactivity Triggered by defective Mitophagy Activates NF-kB Signaling and Causes Neurotoxicity in Frontotemporal Dementia Type 3
}

Abinaya chandrasekaran

Kobenhavns Universitet

Katarina Stoklund Dittlau

KU Leuven: Katholieke Universiteit Leuven

Giulia Corsi

Kobenhavns Universitet

Henriette Haukedal

Kobenhavns Universitet

Nadezhda Doncheva

Kobenhavns Universitet

Sarayu Ramakrishna

Institute for Stem Cell Science and Regenerative Medicine

Scheetal Ambardar

Institute for Stem Cell Science and Regenerative Medicine

Claudia Salcedo

Kobenhavns Universitet

Sissel Schmidt

Odense University: Syddansk Universitet

Yu Zhang

Lunds Universitet

\section{Susanna Cirera}

Kobenhavns Universitet

Benjamin Schmid

Bioneer A/S

Troels Tolstrup Nielsen

University of Copenhagen

Jørgen Erik Nielsen

University of Copenhagen

Miriam Kolko

Kobenhavns Universitet

Julianna Kobolak 
BioTalentum Ltd

\section{Andras Dinnyes}

BioTalentum Ltd

\section{Poul Hyttel}

Kobenhavns Universitet

\section{Dasaradhi Palakodeti}

inStem: Institute for Stem Cell Science and Regenerative Medicine

\section{Jan Gorodkin}

Kobenhavns Universitet

\section{Ravi Muddashetty}

inStem: Institute for Stem Cell Science and Regenerative Medicine

\section{Morten Meyer}

Odense University: Syddansk Universitet

\section{Blanca Irene Aldana}

Kobenhavns Universitet

Kristine Freude ( $\square$ kkf@sund.ku.dk)

Kobenhavns Universitet https://orcid.org/0000-0001-9480-2386

\section{Research article}

Keywords: CHMP2B FTD3, hiPSC-derived astrocytes, autophagy, mitochondria, cytokines

Posted Date: March 16th, 2021

DOI: https://doi.org/10.21203/rs.3.rs-310167/v1

License: (c) (1) This work is licensed under a Creative Commons Attribution 4.0 International License.

Read Full License 


\section{Abstract}

Background: Frontotemporal dementia type 3 (FTD3) caused by a point mutation in the charged multivesicular body protein 2B (CHMP2B), affects mitochondrial ultrastructure and function as well as endosomal-lysosomal fusion in neurons. However, there is a critical knowledge gap in understanding how mutations in CHMP2B affect astrocytes. Hence, we investigated the disease mechanisms in astrocytes derived from hiPSC with mutations in CHMP2B and their impact on neurons.

Methods: To dissect the astrocyte-specific impact of mutant CHMP2B expression, we generated astrocytes from human induced pluripotent stem cells (hiPSCs) from FTD3 patients and their CRISPR/Cas 9 gene edited isogenic controls and produced heterozygous and homozygous CHMP2Bmutant hiPSC via CRISPR/Cas 9 knock-in gene editing. Additionally, we confirmed our findings in CHMP2B mutant mice. The hiPSC were subjected to astrocyte differentiation and the mutation dependent effects were investigated using immunocytochemistry, western blot, cytokine assays, transmission electron microscopy, RNA-sequencing and gas chromatography-mass spectrometry. Finally, neurons were exposed to conditioned media of mutant astrocytes and viability, growth and motility were measured.

Results: To dissect the astrocyte-specific impact of mutant CHMP2B expression, we generated astrocytes from human induced pluripotent stem cells (hiPSCs) and confirmed our findings in CHMP2B mutant mice. Our findings include perturbed mitochondrial dynamics with impaired glycolysis, increased reactive oxygen species and elongated mitochondrial morphology, indicating increased mitochondrial fusion in FTD3 astrocytes. Furthermore, we identified a shift in astrocyte homeostasis triggering a reactive astrocyte phenotype and increased release of toxic cytokines. This cumulates in NF-kB pathway activation with increased production of CHF, LCN2 and C3, which cause neurodegeneration. The neurotoxic effect was investigated by exposing hiPSC-derived neurons to astrocyte-conditioned media, which severely reduced neurite outgrowth capacities. Rescue experiments targeting ROS could restore ROS levels back to normal levels, indicating that the impaired removal of abnormal mitochondria triggers the pathological cascade in CHMP2B mutant astrocytes culminating in the formation of neurotoxic reactive astrocytes.

Conclusion :Our data provide mechanistic insights into how defective mitophagy causes impaired mitochondrial fission, leading to the adoption of reactive astrocyte properties with increased cytokine release, NFkB activation and elevated expression of neurotoxic proteins in FTD3.

\section{Full Text}

Due to technical limitations, full-text HTML conversion of this manuscript could not be completed. However, the latest manuscript can be downloaded and accessed as a PDF.

\section{Figures}


Figure 1

a

Induction

b
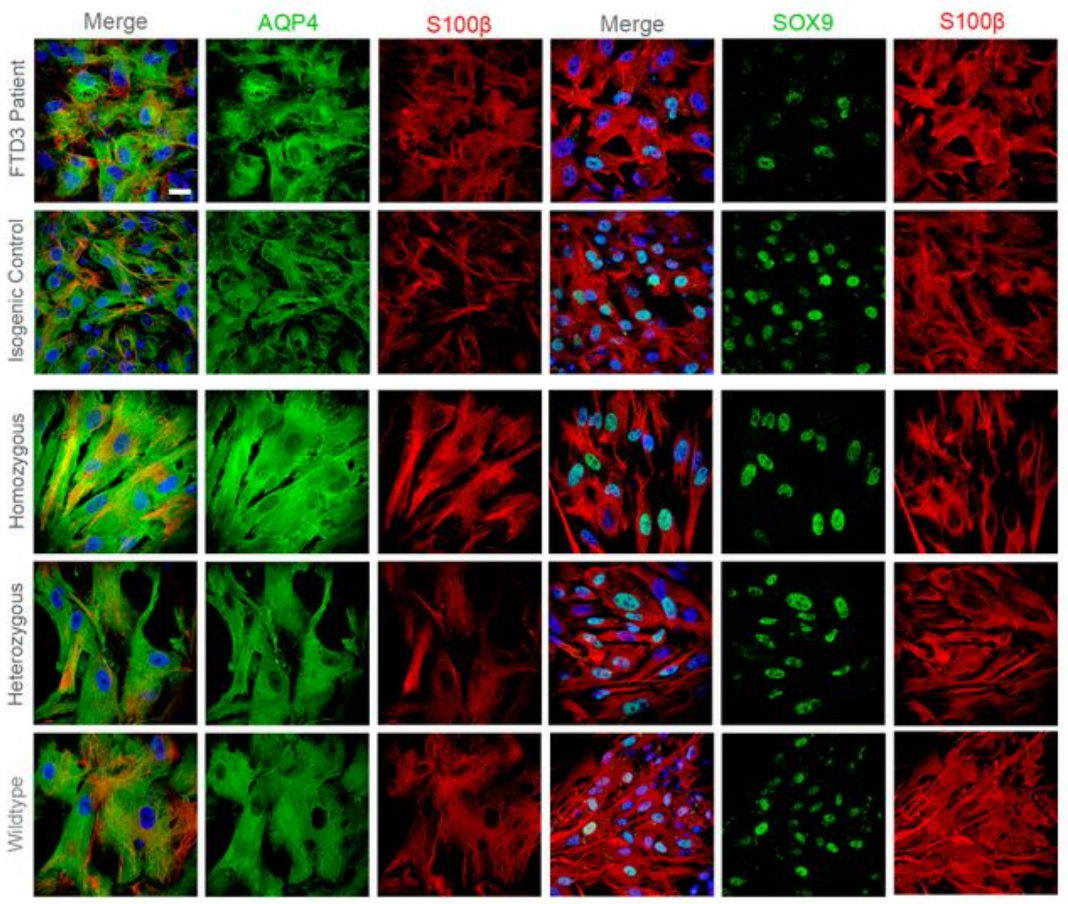

C

d
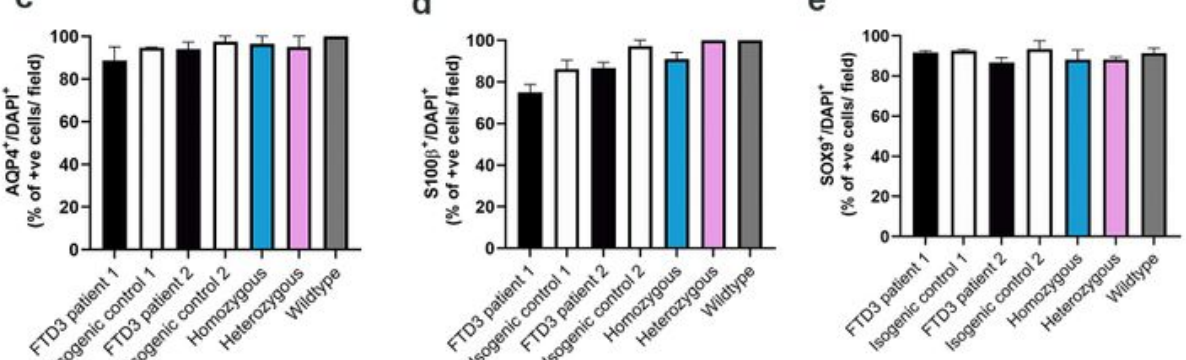

\section{Figure 1}

Generation and Characterization Of hiPSC-derived Astrocytes. a) Schematic of astrocyte differentiation protocol. b) Representative ICC images of AQP4, S100 $\beta$ and SOX9. Scale bar= $25 \mu \mathrm{m}$. c-e) Quantifications of AQP4, S100 $\beta$ and SOX9 via ICC. Data reported as mean \pm SEM, (one-way ANOVA, Tukey multiple comparisons test; $\left.{ }^{*} p<0.05\right)$. $N=3, n=3, N$ indicates the number of experimental repetitions; $\mathrm{n}$ indicates the number of technical replicates per cell line. 


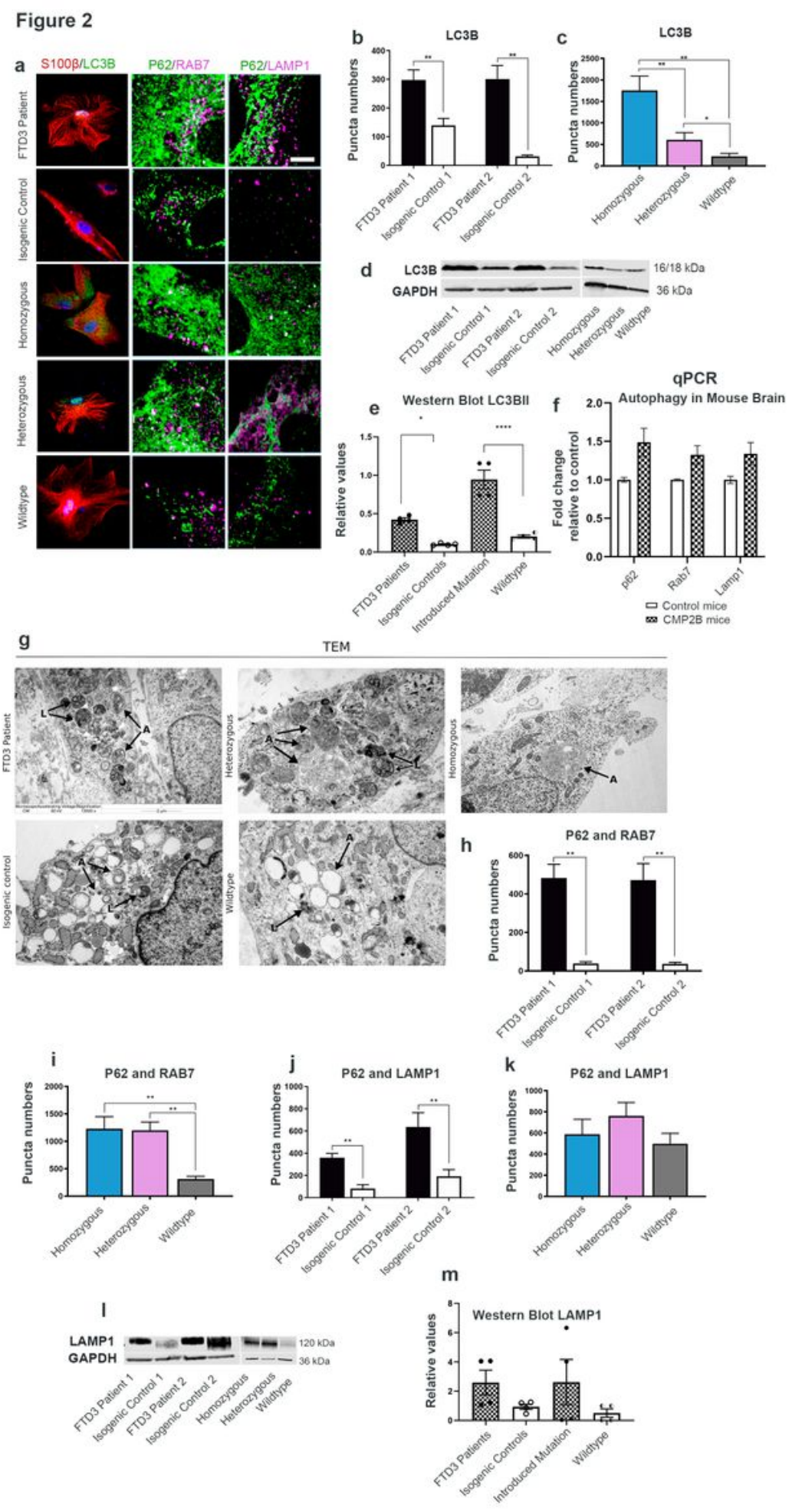

Figure 2

FTD3 Astrocytes Display Autophagy Changes. a) Representative images of LC3B and S100 $\beta$ co-labelling of astrocytes (left column), P62 and RAB7 co-labelling of astrocytes (middle column) and P62 and LAMP1 co-labelling of astrocytes (right column) Scale bar= $25 \mu \mathrm{m} . \mathrm{b}, \mathrm{c}$ ) LC3B quantification. d-e) Western blot of LC3B. f) Validation of CHMP2Bintron5 mice vs. Control mice brain for Autophagy. g) Qualitative ultrastructural visualizion of FTD3-specific accumulation of electon-dense autophagosomes by TEM 
indicated with black arrows. ( $A=$ autophagosomes and $L=$ autolysosomes $\mathrm{h}-\mathrm{i}$ ) Puncta quantifications of P62 and RAB7 colocalization. j-k) Puncta quantifications of P62 and LAMP1 colocalization I-m) Western blot of LAMP1. Data reported as mean \pm SEM, (one-way ANOVA, Tukey multiple comparisons test; ${ }^{*} p<0.05,{ }^{* *} p<0.01$ and $\left.{ }^{* \star *} p<0.001, N=3, n=3\right)$. N indicates the number of experimental repetitions; $n$ indicates the number of technical replicates per cell line. See also Additional Figure S2, S3, S4.

Figure 3

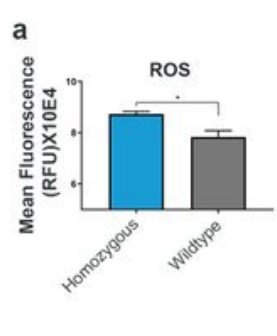

b

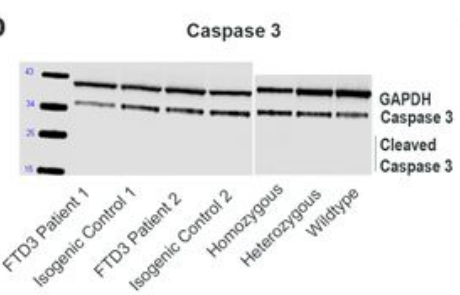

d

Mitotracker
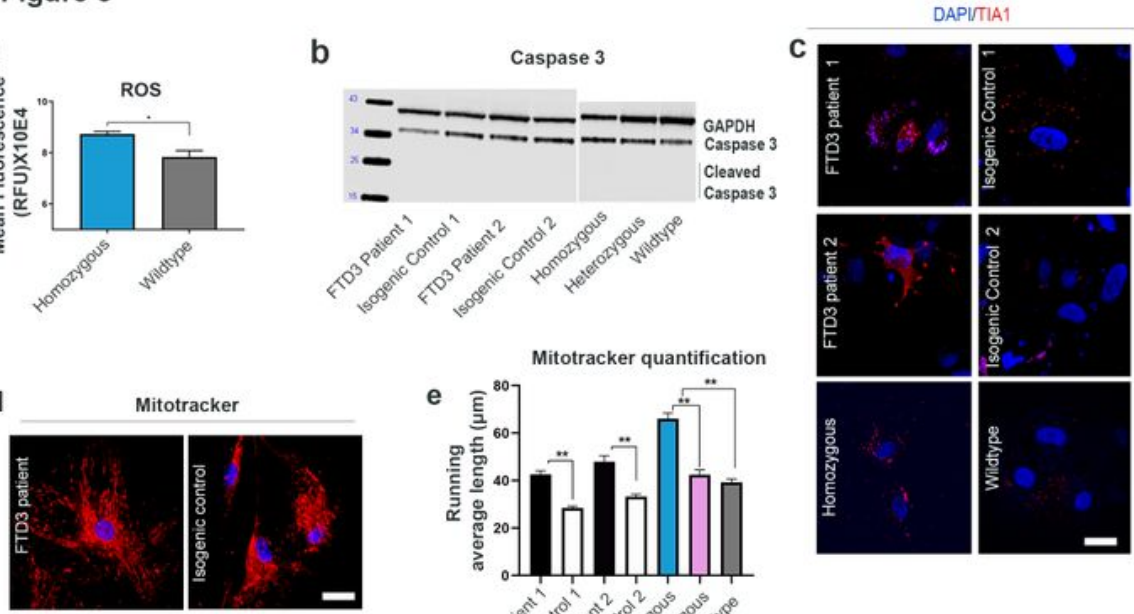

f
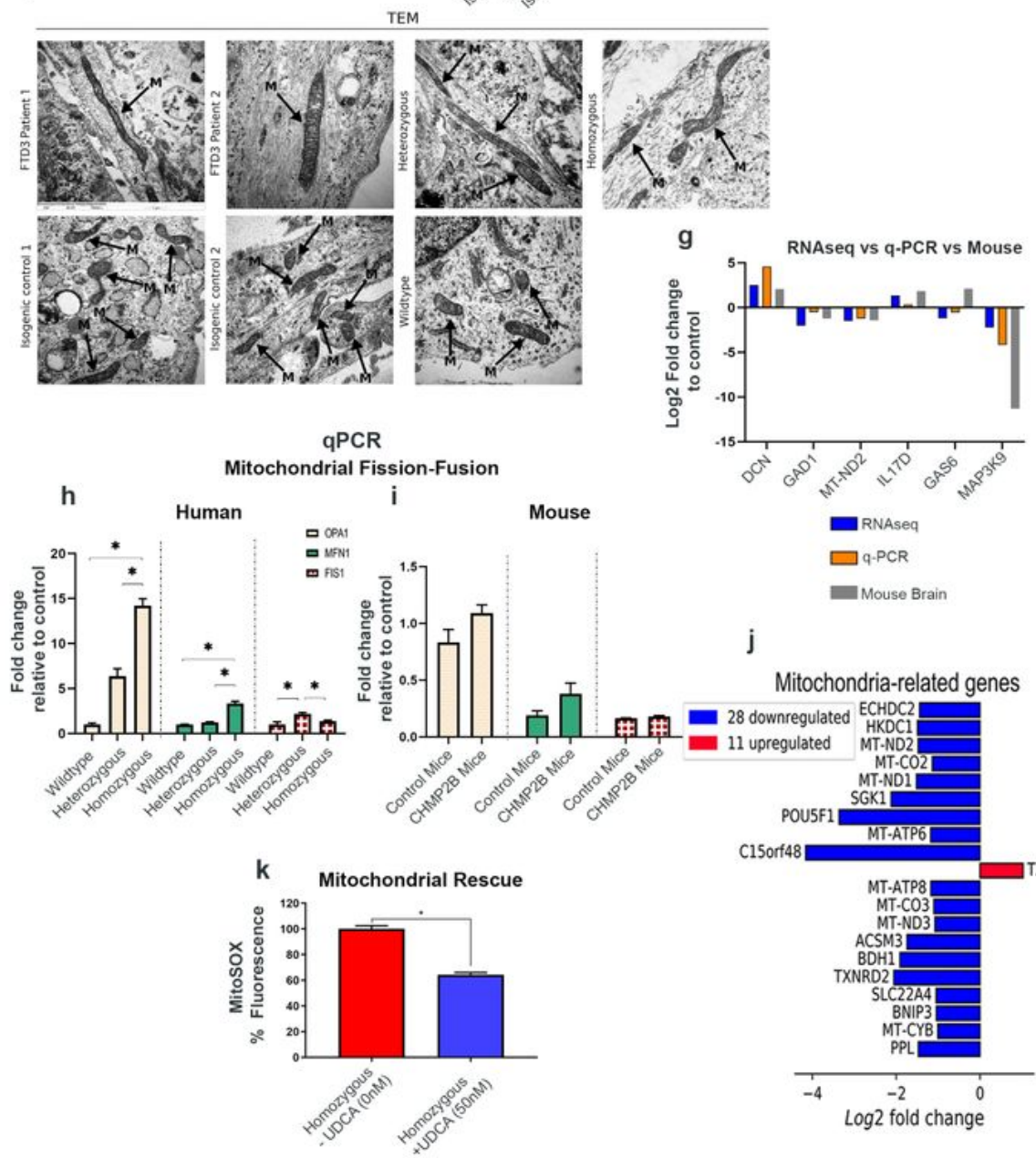

j

Mitochondria-related genes

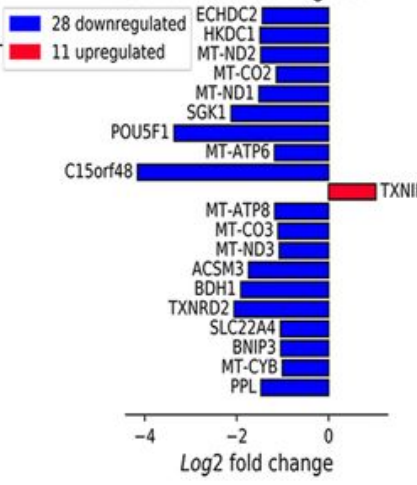

Figure 3 
FTD3 Astrocytes Displays Impaired Mitochondrial Dynamics a) MitoSOX assay b) Caspase 3 activity, GAPDH as control. c) Representative T1A1 labelling, Scale bar $=25 \mu \mathrm{m}$. d) Representative mitochondrial distribution, Scale bar $=25 \mu \mathrm{m}$. e) Running average length of mitochondria. f) Qualitative analysis of mitochondria visualized with TEM. Mitochondria are marked by black arrows. g) RNA-seq validated by qPCR h-i) qPCR determining the fission and fusion genes (OPA1, MFN1, and FIS1). j) Top 20 significantly differentially expressed genes associated with mitochondria. $\mathrm{k}$ ) Rescue of mitochondrial function by treatment with $50 \mathrm{nM}$ UDCA for $24 \mathrm{~h}$. Data are reported as mean \pm SEM. Statistics for RNAseq used Benjamin-Hochberg adjusted P-value (Wald test) $<=0.05$, absolute log2 fold change $>=1$ and mean of normalized counts $>=10$, qPCR used unpaired student t-test and for remaining analyses one-way ANOVA, Tukey multiple comparisons test, $(* p<0.05, * \star p<0.01$ and $* \star \star x p<0.001)$. Significant differences are indicated by $* p<0.05) . N=3, n=3, N$ indicates the number of experimental repetitions; $n$ indicates the number of technical replicates per cell line. See also Additional Figure S1. 
Figure 4
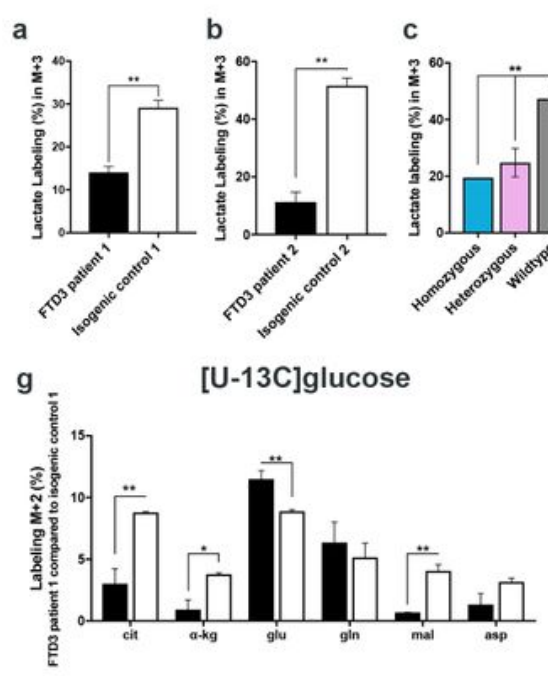

h

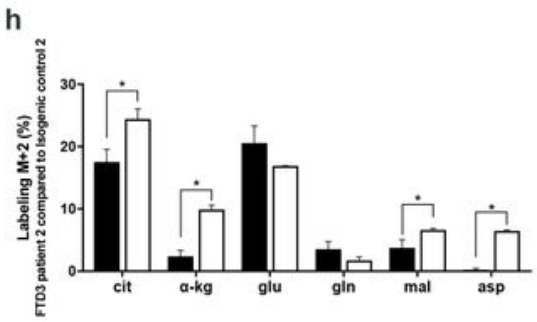

i

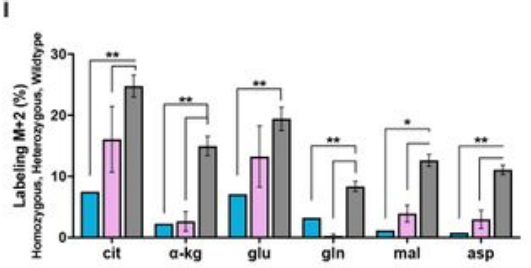

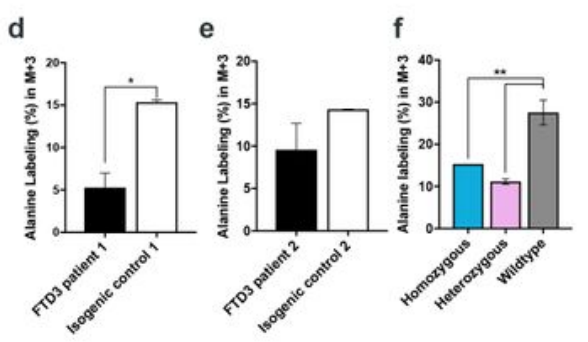

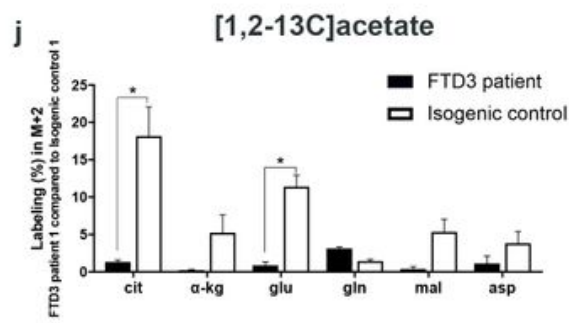

k

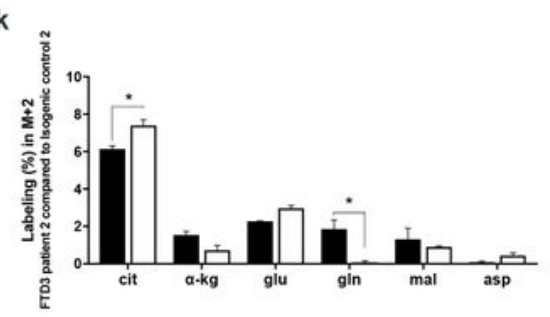

I

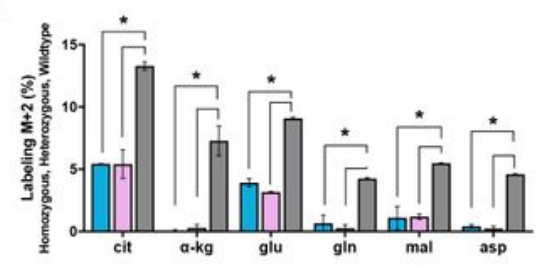

m

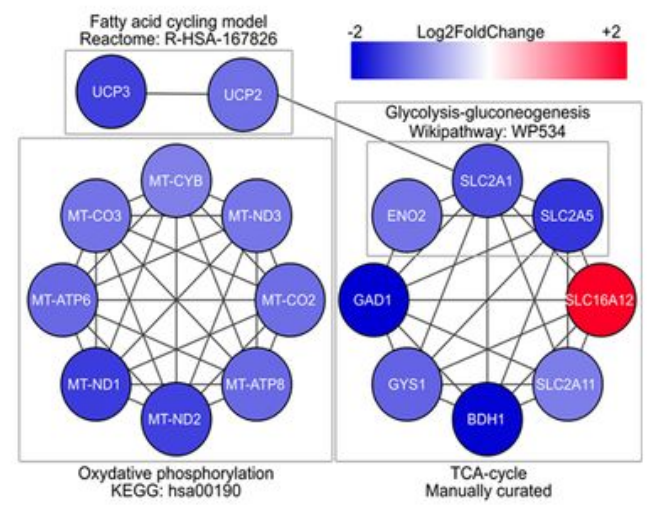

Figure 4

Energy Hypometabolism in FTD3 Astrocytes. a-i) Glucose metabolism: 13C-enrichment in metabolites was determined via GC-MS after 90-min incubation with [U-13C] glucose. j-I) Acetate metabolism. m) Networks of down-regulated genes involved in energy-production pathways. Node colors represent expression fold changes in a blue-white-red logarithmic scale. Results are reported as mean \pm SEM, (twoway ANOVA with Tukey multiple comparisons test; ${ }^{*} p<0.05$, $\left.{ }^{* *} p<0.01\right) . N=3, n=3, N$ indicates the number 
of experimental repetitions; $\mathrm{n}$ indicates the number of technical replicates per cell line. See also Additional Figure S1.

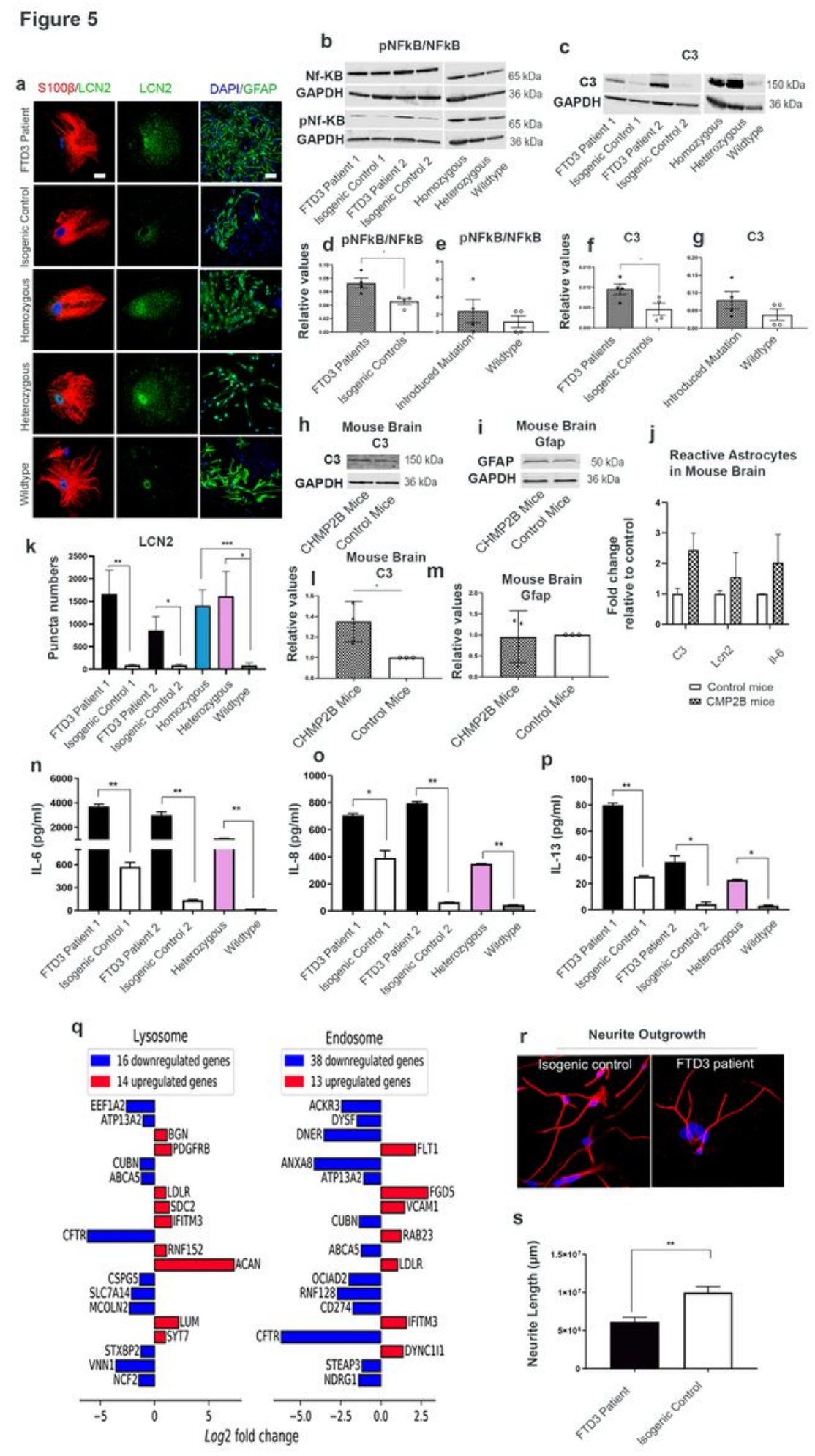

Figure 5

FTD3 Pathology Contributes To Reactive Astrocyte. a) Representative images of LCN2 and S100 $\beta$ colabelling of astrocytes (left column) and GFAP labelling of astrocytes (right column). Scale bar $=25 \mu \mathrm{m} . \mathrm{b}$, d, e) Western blot of Nf-KB and pNf-KB. c, f, g) Western blot of C3 (human). h, I) Western blot of C3 for 
CHMP2Bintron5 mice brain $\mathrm{I}, \mathrm{m}$ ) Western blot of Gfap (mouse). $\mathrm{j}$ ) Validation of CHMP2Bintron5 mice brain for reactive astrocytes. k) LCN2 puncta quantification. n-p) Cytokine profiling. q) Top 20 significantly differentially expressed genes associated with lysosomes (left) and endosomes (right). r) Representative image of neurite outgrowth. s) Data analysis of neurite outgrowth. Data are reported as mean \pm SEM, (one-way ANOVA, Tukey multiple comparisons test; ${ }^{*} p<0.05,{ }^{* \star} p<0.01$ and ${ }^{\star \star \star} p<<0.001$ ). $\mathrm{N}=3, \mathrm{n}=3, \mathrm{~N}$ indicates the number of experimental repetitions; $\mathrm{n}$ indicates the number of technical replicates per cell line. See also Additional Figure S1, S6.

\section{CHMP2B mutation}

\section{Autophagy and mitophagy impairment}
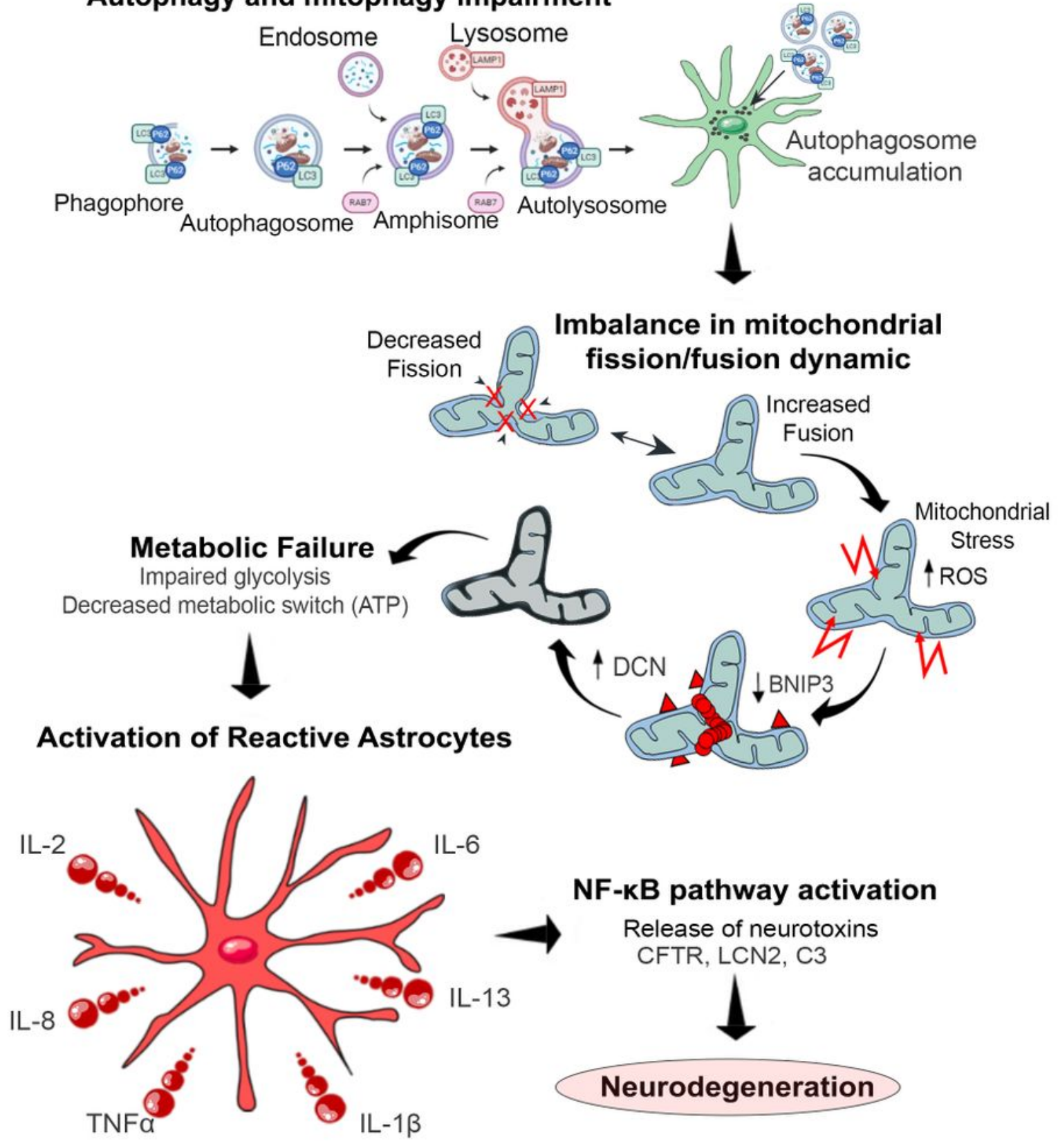
Figure 6

Proposed model: Role of Astrocytes in FTD3 pathology Mutant CHMP2B impairs normal mitophagy, leading to accumulation of elongated and dysfunctional mitochondria triggering oxidative stress. These cascades of events culminate in conversion of resting astrocytes to reactive astrocytes releasing neurotoxic cytokines, contributing to neuronal dysfunction and apoptosis in FTD3.

\section{Supplementary Files}

This is a list of supplementary files associated with this preprint. Click to download.

- Supplementerylnformation.pdf

- SupplementFigureS1.jpg

- SupplementFigureS2.jpg

- SupplementFigureS3.jpg

- SupplementFigureS4.jpg

- SupplementFigureS5.jpg

- SupplementFigureS6.jpg

- SupplementaryTables2.xls

- SupplementaryTableS3.xls 